\title{
Delimitación y descripción biofísica de dos turberas y de una laguna endorreica en el sector Salsipuedes-Cerro Asunción, Parque Nacional Tapantí - Macizo de la Muerte, Costa Rica
}

\author{
Yazmín León Alfaro' \& Jonnathan Reyes Chaves ${ }^{2}$ \\ 1. Programa de Doctorado en Ciencias Humanas: Geografía, Antropología y Estudios de África y Asia, Departamento de Geografía, Universidad \\ Autónoma de Madrid, Madrid, España; yazmin.leon@predoc.uam.es \\ 2. Escuela de Geografía, Universidad de Costa Rica, San José, Costa Rica; jonnathan.reyeschaves@ucr.ac.cr
}

Recibido 22-VIII-2017 • Corregido 13-XI-2017 • Aceptado 30-XI-2017

\begin{abstract}
Delimitation and biophysical description of two peat bogs and an endorheic lagoon in Salsipuedes-Cerro Asunción, Tapantí - Macizo de la Muerte National Park, Costa Rica. Recognition of peatlands as an essential part of global wetland resources has increased in recent years. Roughly half of the world's wetlands are peatlands and are particularly vulnerable to human activities and climate change. We present the first a biophysical description of vegetation, micro relief and water of peat bogs in Macizo de la Muerte National Park, Costa Rica. Three field transects, and LANDSAT 8 images, indicate that bogs differ in water table trends and vegetation. One bog had a central flooded area dominated by Sphagnum spp.; a transitional sector with Puya dasylirioides, Blechnum buchtienii and Chusquea subtessellata; and a shrub layer dominated by Pernettya prostrata and Vaccinium consanguineum. Most trees were Quercus seemanni and Drymis granadensis. Another bog was characterized by Puya dasylirioides, Blechnum buchtienii and the aquatic herbs Paepalanthus costaricensis and Hypericum strictum, trees included Quercus seemannii and Drymis granadensis (at the edges of the wetland). The progressive reduction of water in wetlands represents a risk of degradation and transformation of bogs into another type of ecosystem.
\end{abstract}

Key words: Peat bogs, endorheic lagoon, vegetation structure, micro reliefs, water table, Tapantí-Macizo de la Muerte National Park.
RESUMEN: El reconocimiento de las turberas como parte esencial de los recursos humedales del mundo ha aumentado en los últimos años. Aproximadamente la mitad de los humedales del mundo son turberas y son particularmente vulnerables a las actividades humanas y el cambio climático. Presentamos la primera descripción biofísica de la vegetación, el micro relieve y el agua de turberas en el Parque Nacional del Macizo de la Muerte, Costa Rica. Tres transectos y las imágenes de LANDSAT 8 indican que las turberas difieren en las tendencias de la capa freática y la vegetación. Una turbera tenía una zona central inundada dominada por Sphagnum spp.; un sector de transición con Puya dasylirioides, Blechnum buchtienii y Chusquea subtessellata; y una capa de arbustos dominada por Pernettya prostrata y Vaccinium consanguineum. La mayoría de los árboles eran Quercus seemanni y Drymis granadensis. Otra turbera se caracterizó por Puya dasylirioides, Blechnum buchtienii y las hierbas acuáticas Paepalanthus costaricensis e Hypericum strictum, incluidos los árboles Quercus seemannii y Drymis granadensis (en los bordes del humedal). La reducción progresiva del agua en los humedales representa un riesgo de degradación y transformación de las turberas en otro tipo de ecosistema.

Palabras clave: Turberas, laguna endorreica, estructura vegetal, microrelieves, tabla de agua, Parque Nacional Tapantí-Macizo de la Muerte.
La turba está formada por restos de plantas muertas y parcialmente descompuestas que se han acumulado in situ en terrenos anegados, por lo cual son ecosistemas con alto contenido de materia orgánica que desempeñan funciones ambientales vitales. El reconocimiento de las turberas como parte esencial de los recursos mundiales de humedales ha aumentado en los últimos años, ya que aproximadamente la mitad de los humedales a nivel mundial son turberas, encontrándose desde zonas boreales hasta tropicales (Secretaría de la Comisión de Ramsar, 2007). La gran diversidad que presentan las turberas, dependiendo del clima, la topografía, la geomorfología y la hidrología ha generado vastas investigaciones registradas desde 1762 con los estudios de Francis Home, sobre destilación de ácidos orgánicos en la turba (Pontevedra, 2001).

Los países de latitudes templadas con amplias extensiones de turberas y suelos con características turbosas 
desarrollaron diversas clasificaciones, utilizando criterios como hidrología, relieve, vegetación, propiedades químicas, morfología y procesos genéticos, entre otras. Nuevas interrogantes plantearon la necesidad de ahondar en el conocimiento de los diversos elementos del ecosistema. Siguiendo a Pontevedra (2001), en los últimos años, y probablemente de cara al futuro, los conocimientos adquiridos sobre el funcionamiento y naturaleza de las turberas convergen hacia tres líneas de investigación principales: 1) la delimitación cartográfica, geográfica e inventarios de los ecosistemas de turberas a nivel mundial, para su conservación y restauración, 2) el estudio de la evolución climática para comprender origen y desarrollo de estos medios, y 3) el seguimiento del deterioro medioambiental global utilizando la reconstrucción temporal de la composición atmosférica, esto a través del análisis del contenido en elementos traza de turberas ombrotróficas y las tasas de producción de gases de efecto invernadero. Es en la primera línea que se enmarca el presente estudio como un aporte para el inventario de estos humedales en Costa Rica.

La Cordillera de Talamanca alberga humedales de turbera, específicamente en el Parque Nacional Chirripó, la Reserva Forestal Los Santos, Parque Nacional TapantíMacizo de la Muerte y Parque Internacional La Amistad (Programa Nacional de Humedales SINAC-MINAE, 2002) . Estos forman parte del ecosistema boscoso más grande de Costa Rica, que constituye un inmenso corredor biológico que posibilita el desplazamiento altitudinal y longitudinal de especies de flora y fauna.

Estas turberas, según Brak, Vroklage, Kappelle y Cleef (2005), fueron estudiadas inicialmente mediante descripciones de Weber (1958), en el sitio Madre Selva, Gómez (1984, 1986) y por Augspurger (1985), en el Cerro de la Muerte. Weston (1981), según estos autores, definió este ecosistema como ciénaga e identificó sus suelos como mal drenados y ácidos. Según ese autor, la mayor extensión de turberas se encuentra en las sabanas de Dúrika y en la zona de Utyum, donde existen comunidades dominadas por el helecho Lomaria (Blechnum), la bromelia Puya dasylirioides y las hierbas acuáticas Paepalanthus costaricensis y Xyris, todas asociadas con el musgo Sphagnum spp.

Por otra parte, indican que Gómez (1986), comparó las turberas de altura con los páramos, por su gran similitud florística, y con las turberas o charcas permanentes de las sabanas de bajura, por su similitud fisonómica y ecológica. Este autor identificó la presencia de los géneros vasculares Blechnum, Puya, Chusquea, Rapanea (Myrsine) y Vaccinium, asociados al género de briófitas Sphagnum y a una alga roja del género Batrachospermum. Reporta, también, que las aguas de las turberas son de reacción ácida y que se desarrollan sobre depresiones entre cerrillos y colinas. Hooghiemstra, Cleef, Noldus y Kappelle (1992) estudiaron la paleoecología y la palinología de las turberas, o "peat bogs", de La Chonta y La Trinidad de Dota, a lo largo de la Carretera Interamericana Sur.

Fuera de la Cordillera de Talamanca Obando y Soto (1993) estudiaron la turbera de río Silencio, en El Cairo, Siquirres, estableciendo una correlación entre los horizontes de ceniza en esa turbera y las erupciones del volcán Turrialba. Por su parte, los citados Brak et al. (2005), estudiaron nuevamente la turbera La Chonta, identificando y cartografiando las comunidades vegetales de dicha turbera.

Los estudios realizados han contribuido a la comprensión de las turberas como ecosistemas muy vulnerables ante perturbaciones humanas y cambios ambientales. En concordancia con esto, el presente estudio se centra en el objetivo de delimitar dos turberas y una laguna endorreica en el sector Salsipuedes y Cerro Asunción, en el Parque Nacional Tapantí-Macizo de la Muerte, así como describir la estructura de la vegetación y la relación de ésta con la variación de la topografía, y con la tabla de agua en el suelo, con el fin de aportar datos iniciales para su futuro monitoreo.

\section{MATERIALES Y MÉTODOS}

Caracterización del área de estudio: La zona de estudio se localiza en la Cordillera de Talamanca, dentro del Parque Nacional Tapantí-Macizo de la Muerte, en este último sector, comprendiendo dos turberas ubicadas en la margen derecha de la carretera Interamericana Sur, en dirección a la zona conocida como Salsipuedes y una laguna endorreica ubicada al pie del Cerro Asunción (Fig. 1 en Apéndice Digital). La turbera uno se localiza según las coordenadas geográficas $9^{\circ} 39^{\prime} 41^{\prime \prime} \mathrm{N}$ y $80^{\circ} 50^{\prime} 55^{\prime \prime} \mathrm{W}$, con una altitud promedio de 2670 m.s.n.m. La turbera dos se localiza según las coordenadas $9^{\circ} 39^{\prime} 09^{\prime \prime} \mathrm{N}$ y $80^{\circ} 50^{\prime} 42^{\prime \prime} \mathrm{W}$, con una altitud promedio de 2767 m.s.n.m. Por su parte, la laguna endorreica en el sector Cerro Asunción está comprendida en las coordenadas $9^{\circ} 39^{\prime} 09^{\prime \prime} \mathrm{N}$ y $80^{\circ} 50^{\prime} 42^{\prime \prime}$ W, con una altitud promedio de 3335 m.s.n.m.

Las turberas se ubican en el piso altitudinal de los bosques montanos bajos y altos, pero presentando una similitud estructural y florística con la vegetación de páramos. La vegetación presenta adaptaciones a condiciones extremas, estacionalidad climática y alta radiación solar (Programa Nacional de Humedales SINAC-MINAE, 2002). El suelo es ácido y saturado de agua la mayor parte del tiempo, lo que reduce la descomposición de 
la materia orgánica muerta, causando la acumulación de turba. Las características pedológicas y topográficas, junto con las bajas temperaturas y altas precipitaciones entre 1800 a $3000 \mathrm{~mm}$, propias de un clima húmedo a muy húmedo, templado a frío, permiten el desarrollo de este ecosistema.

El estudio partió de la delimitación en campo de la extensión de las turberas y la laguna mediante un receptor GPS (Global Positional System), con una precisión de +/-3m. La descripción estructural de la vegetación permitió también determinar la extensión de las turberas diferenciándolas de las formaciones boscosas zonales que las bordean. Dicha descripción se realizó a través de transeptos evidenciando un perfil topográfico para identificar la variabilidad de microrelieves y de la tabla de agua en el suelo.

El trabajo de campo se concentró en la turbera uno, ya que dada su mayor variabilidad biofísica se tomó como sitio inicial para implementar la metodología propuesta. El transepto se llevó a cabo transversalmente con una longitud de $67,7 \mathrm{~m}$ y se realizó durante una salida de campo en noviembre de 2014, donde se trabajó cuatro segmentos del mismo con las siguientes medidas: $17,8 \mathrm{~m}, 7,50 \mathrm{~m}$, $7,9 \mathrm{~m}$ y $8,7 \mathrm{~m}$. Los restantes segmentos no se trabajaron debido al difícil acceso por la densidad de la vegetación briófita en montículos. Se ató una cuerda con estacas distanciadas a una altura de $1 \mathrm{~m}$ del suelo y las mediciones de la topografía se realizaron cada $50 \mathrm{~cm}$ desde el alto de la cuerda al terreno. A lo largo de los segmentos se localizó la vegetación mediante un sistema de coordenadas $X$ y Y, registrándose la vegetación en una banda de $50 \mathrm{~cm}$ a los lados de la cuerda. Las especies fueron descritas solo estructuralmente, cuando no se conocía con certeza el nombre científico a nivel de especie. Así mismo, a lo largo de los segmentos del transepto fueron registradas, mediante fotografías, observaciones sobre evidencia de saturación del suelo por encharcamiento.

En la turbera dos se realizaron dos transeptos de $14 \mathrm{~m}$ de longitud cada uno durante visita de campo efectuada en noviembre de 2015. Estos contaron con anotaciones puntuales sobre la estructura vegetal y su distribución en un sistema de coordenadas $X$ y $Y$.

En el caso de la laguna endorreica fueron levantados datos iniciales para la elaboración de una batimetría mediante un sondeo con varillas. Adicionalmente, se realizó una clasificación supervisada de las coberturas en el entorno geográfico de las turberas mediante el procesamiento de una imagen LANDSAT 8 , con una resolución espacial de $30 \mathrm{~m}$ por píxel, esto para la determinación de la extensión de las turberas y de la laguna. Fueron seleccionados pixeles de la imagen con valores afines a una potencial categoría de "turberas", es decir la selección de áreas de entrenamiento que permitieran extraer tal categoría.

\section{RESULTADOS}

La turbera uno cuenta con una forma alargada (Fig. 2 en Apéndice Digital) presentando las siguientes dimensiones: $119,4 \mathrm{~m}$ punto más ancho, $338,3 \mathrm{~m}$ de longitud y un perímetro de $998,8 \mathrm{~m}$. Por su parte, la turbera dos tiene forma más ovalada, pero con una prolongación hacia el sur (Fig. 3 en Apéndice Digital), con dimensiones de $13,3 \mathrm{~m}$ en el punto más ancho y $22,7 \mathrm{~m}$ de longitud. Los resultados encontrados en las turberas según lo observado en los transeptos evidencian la relación entre la variación topográfica y el contenido de humedad con la estructura de la vegetación, principalmente en la turbera uno, la cual fue tomada como estudio piloto.

Turbera uno: En cuanto a la variación topográfica en la turbera uno se identificó una inclinación pronunciada del terreno hacia los bordes de ésta, con presencia de una zona depresional central, generando una morfología cóncava cerrada. Esta zona no presenta un relieve homogéneo, sino que hay microrelieves con forma de pequeños montículos y sitios más bajos, con secciones anegadas, que se encharcan de forma más o menos permanente., menores a los $5 \mathrm{~cm}$ de profundidad y grietas que captan grandes cantidades de agua superiores a $50 \mathrm{~cm}$ de profundidad. Se observaron también pequeños canales que corresponden al movimiento de flujos de agua superficial dentro de la turbera. Fue observado, así mismo, un drenaje construido en dirección oeste hacia la carretera Interamericana Sur (Fig. 4 en Apéndice Digital).

En cuanto a la distribución de la vegetación en relación con la variación topográfica y la tabla de agua en el suelo (Fig. 5 en Apéndice Digital), se distinguió tres secciones diferenciadas: una zona central con espejos de agua dispersos y con predominio de musgo del género Sphagnum y hierbas acuáticas como Paepalanthus costaricensis, Hieracium irazuense e Hypericum strictum; un sector de transición con pendientes más altas, menor encharcamiento y presencia de la bromelia terrestre Puya dasylirioides y de helechos arborescentes Blechnum buchtienii, así como de Chusquea subtessellata (Fig. 6 en Apéndice Digital). La especie Cortaderia nítida se encontró formando macollas en los límites con la tercera sección. Ésta última presentó dos estratos, uno arbustivo, representado principalmente por Pernettya prostrata y Vaccinium consanguineum, y otro arbóreo, principalmente Quercus seemanni y Drymis granadensis. 
Turbera dos: La turbera dos del sector Salsipuedes se caracteriza por una topografía predominantemente plana, con pocos cambios en el relieve. Comparativamente con la turbera uno y en proporción a su área, ésta presenta más superficie en estado de anegamiento. En algunos sectores, donde la tabla de agua es reducida, se pudo observar afloramientos rocosos con suelos poco desarrollados. A diferencia de la turbera uno, la presencia de montículos y pequeñas zonas bajas es más reducida. A nivel de especies vegetales, se encuentran fundamentalmente las mismas especies encontradas en la turbera uno, como Puya dasylirioides y el helecho Blechnum buchtienii, además del predominio en los sectores anegados con plantas vasculares como Paepalanthus costaricensis e Hypericum strictum (Figs. 7 y 8 en Apéndice Digital).

Laguna endorreica y coberturas en el área estudiada: Por su parte, en la laguna endorreica del Cerro Asunción (Fig. 9 en Apéndice Digital), fueron identificados dos niveles de terrazas que se elevan desde el espejo de agua actual a un metro el primero y un metro con $35 \mathrm{~cm}$ el segundo (Fig. 10 en Apéndice Digital). La profundidad máxima levantada en campo fue de $52 \mathrm{~cm}$, esto en época seca (Fig.11 en Apéndice Digital). Los resultados de la clasificación de coberturas (Fig.12 en Apéndice Digital), donde se presentan las categorías identificadas, a saber, turberas, nubes, sombra de nubes y otro tipo de vegetación.

\section{DISCUSIÓN}

Las turberas estudiadas corresponden con ecosistemas azonales, ya que su existencia obedece fundamentalmente a características específicas de los suelos y de la geomorfología, no así a las climáticas. La turbera uno se localiza en una depresión topográfica, habitual de turberas tropicales de alta montaña, lo que permite la acumulación de humedad en la parte central y la disminución de la misma hacia los bordes (Benavides, 2013; Jiménez, 2016). Los suelos característicos son histosoles, mal drenados, porosos, con poca disponibilidad de oxígeno, que ocasiona una baja actividad de microorganismos y bajas tasas de mineralización. Esto genera la acumulación de materia orgánica en diferentes grados de descomposición y según los aportes de agua de la atmósfera se manifiesta en $\mathrm{pH}$ ácidos y baja disponibilidad de nutrientes que varían según el gradiente topográfico (Martínez, Pontevedra, Novoa, Rodríguez \& López-Sáez, 2009).

La turbera uno presenta en su zona depresional, una serie de microrelieves del tipo "hummock-hollow" (Brak et al., 2005), correspondiente a pequeños montículos y zonas más bajas cubiertas mayormente por el musgo Sphagnum spp. en condiciones de anegamiento, así como de Eleocharis acicularis. Esta zona con mayor humedad está surcada por delgados canales por donde circula el agua superficialmente, junto con la presencia de pequeñas grietas de un máximo de $5 \mathrm{~cm}$ de profundidad y otras con forma de grandes brechas de hasta $50 \mathrm{~cm}$ llenas de agua, coincidentes con áreas cubiertas por Xyris subulataba y Xyris nigrescens. Sin embargo, la presencia del drenaje, construido con motivo de la construcción de la carretera Interamericana Sur y que dividió en dos partes el área original de la turbera, podría estar vinculada con una menor cantidad de agua en el humedal, ya que este tipo de intervención afecta la continuidad y profundidad de la capa freática.

Una menor saturación de agua conlleva la descomposición de la materia orgánica por aireación de estratos de turba previamente anaerobia, los procesos de mineralización se ven acelerados y se produce una pérdida de masa (Pontevedra, 2001). Además, esta perturbación resulta relevante, ya que en el caso de las turberas tropicales la acumulación de materia orgánica estaría más asociada a las bajas tasas de descomposición debido a las condiciones anaeróbicas, más que a una alta tasa de productividad primaria, esto según lo encontrado por Chimner y Ewel (2005) en turberas de Micronesia. Posteriormente, el proceso puede ser acelerado por el desarrollo de vegetación de tipo arbustivo, que por medio de sus raíces favorecen la aireación del suelo y la percolación de agua a niveles inferiores. Eventualmente el humedal se degrada y se transforma progresivamente en otro tipo de ecosistema (Iturraspe, 2010).

En cuanto a lo observado a través de los transeptos, en ambas turberas fue posible identificar una tendencia de distribución de especies y estructura de la vegetación desde la depresión central a los bordes del humedal, similar a lo encontrado por Brak et al. (2005) en la turbera La Chonta y en un estudio anterior de Hooghiemstra et al. (1992). Esto con un patrón menos acentuado en la turbera dos, debido a que en ésta la diferenciación topográfica es mucho menor y la tabla de agua es más uniforme en toda la extensión de la turbera. Así también, en la turbera dos no se observó perturbaciones por drenajes artificiales y presencia de infraestructura vial que estuvieran alterando la dinámica hídrica dentro del humedal y su relación con la estructura y distribución de la vegetación.

Las zonas identificadas en el transepto de la turbera uno corresponden con un gradiente ecológico que comprende una primera zona en el sector central de la turbera, caracterizado por un suelo mal drenado y parcialmente anegado, donde predomina el musgo 
Sphagnum y la planta herbácea Eleocharis acicularis. El Sphagnum se observó formando una extensa cobertura y agrupado formando diversos montículos junto con Hieraciun irazuense y Paepalanthus costaricensis.

La segunda zona corresponde con el sector de transición donde la pendiente del terreno empieza a elevarse y las condiciones de humedad en el suelo son más reducidas. Especies con mayor estructura de tipo arbustivo y de mayor altura se encontraron en esta zona topográficamente transicional hacia los bordes elevados de la turbera. Se identificó una abundante presencia de la asociación de bromelia endémica Puya dasylirioides y del helecho arborescente Blechnum buchtienii, este último, según Horich (1959), es un posible remanente de antiguas extensiones de páramos que cubrían más extensamente las zonas más elevadas de la Cordillera de Talamanca. Esta vegetación conocida como paramillo se encuentra usualmente en los márgenes de las turberas, tal como lo indican Hooghiemstra et al. (1992). Dicha vegetación zonal presenta formaciones arbustivas de alrededor de $70 \mathrm{~cm}$ a $1 \mathrm{~m}$ de altura, así como la existencia de la gramínea Chusquea subtessellata, un elemento dominante de la vegetación de páramo y que constituye la más común de las asociaciones de plantas zonales encontradas en los valles elevados y laderas de la Cordillera de Talamanca (Horn \& Lynn, 1992; Kappelle \& Horn, 2016).

Por su parte, la especie de gramínea Cortaderia nítida se encontró formando macollas en zonas de suelo más seco y que presentan características ecológicas de efecto borde, al existir un cambio abrupto en la vegetación, relacionada a contrastes en los factores hidrológicos y edáficos, tal como lo encontraron Brak et al. (2005), al estudiar el perfil ecológico en la turbera La Chonta.

La tercera zona se ubica en los bordes de la turbera, que corresponden a las partes más elevadas del terreno, donde se ha desarrollado una vegetación con un estrato arbustivo Pernettya prostrata, Vaccinium consanguineum, y otro de porte arbóreo, presentando robles o encinos (Quercus seemanni), así como Drymis granadensis de la familia Winteraceae. Estos corresponden con una vegetación de tipo zonal conformada por robles, propia del bosque montano alto, entre los 2400 a 3 300m.s.n.m. (Kappelle, 1996). A partir de estos aspectos se identificó la diferenciación de áreas con vegetación azonal, es decir de turbera, determinada por las condiciones de saturación de agua en el suelo, junto con la variación topográfica del área perimetral más elevada donde se localiza la vegetación zonal que está fuertemente vinculada a condiciones climáticas y por consiguiente a la altitud (Hooghiemstra et al., 1992). Dicha distinción resultó el criterio fundamental en campo para determinar la extensión del humedal.

No obstante, resulta de importancia resaltar que aquí la progresión de dicha vegetación boscosa es más acentuada que en la turbera dos, por lo que serían necesarios estudios de las características del suelo, en términos de contenido de humedad asociado a procesos de acumulación de materia orgánica y de mineralización vinculados a la variación topográfica (Martínez et al., 2009).

La turbera dos presentó una topografía más plana y uniforme, siendo conformada por zonas cubiertas de agua por completo, con mayor predominio de Sphagnum y hierbas acuáticas, fundamentalmente Paepalanthus costaricensis, Hieracium irazuense e Hypericum strictum, donde ocurren procesos lentos de drenaje y de descomposición de la materia orgánica. Puya dasylirioides y el helecho Blechnum buchtienii fueron observados con amplio predominio y mayor presencia hacia los bordes de la turbera, así como ejemplares del pasto Cortaderia nítida y de los arbustos de la familia Ericaceae Pernettya prostrata y Vaccinium consanguineum en el perímetro del humedal. La distribución de la vegetación en la turbera dos mostró un patrón menos marcado de gradiente ecológico, relacionado a una mayor uniformidad topográfica y en la tabla de agua.

Respecto al trabajo realizado en la laguna los datos levantados constituyeron un primer aporte para su monitoreo, mismo que requiere ser continuado con el fin de estudiar si existen procesos iniciales de formación de turba por acumulación de restos vegetales en el fondo acuático (Martínez et al., 2009). Estos podrían ser aportados por la vegetación circundante, cuyas especies corresponden con la formación vegetal de páramo.

La tabla de agua superficial o la propia cobertura vegetal de páramo constituyeron factores de confusión en la identificación de las turberas, por lo que la herramienta de clasificación supervisada, basada en el agrupamiento de pixeles por afinidad de valores, se mostró efectiva para separar los espacios de turberas del resto de coberturas. Sin embargo, la delimitación con mayor precisión de su extensión tuvo como limitante la resolución espacial de la imagen LANDSAT 8, de 30m por píxel. Ello impidió un mapeo detallado de la extensión abarcada por las turberas. Para esto, se requeriría imágenes no gratuitas de otros sensores, con mayor resolución espacial.

Los resultados encontrados, fundamentalmente a lo largo del transepto en la turbera uno, evidencian la relación entre la variación topográfica, los microrelieves y la tabla de agua con la estructura y distribución de la vegetación. Es relevante resaltar la progresión de vegetación boscosa zonal en los bordes de la turbera uno, la 
cual es más acentuada que en la turbera dos. Así mismo, la presencia de un drenaje construido en la turbera uno representa una alteración importante en la dinámica hidrológica del humedal, lo cual puede estar contribuyendo a la pérdida de agua. Para comprender mejor estos procesos e interacciones son necesarios estudios más profundos y continuados.

Por su parte, los datos batimétricos obtenidos para la laguna endorreica constituyen una base para dar seguimiento al comportamiento del espejo de agua, lo que sería necesario realizar sistemáticamente en estación seca y lluviosa.

Este trabajo constituyó un primer acercamiento de propuesta metodológica desde la Geografía, para eventualmente realizar estudios analíticos y con disponibilidad de mayores recursos para generar información cuantitativa. Se requiere, en todo caso, implementar un monitoreo de los aspectos indicados, ya que estos humedales presentan riesgo de degradación y conversión en otro tipo de ecosistema, debido a cambios en la dinámica hidrológica y pedológica.

\section{AGRADECIMIENTOS}

Agradecemos a los estudiantes del curso Técnicas de campo en Geografía GF-5002, del segundo semestre de 2014, impartido en la Escuela de Geografía de la Universidad de Costa Rica, por su trabajo en el levantamiento del transepto en la turbera uno.

\section{REFERENCIAS}

Barber, K. (1993). Peatlands as scientific archives of past biodiversity. Biodiversity And Conservation, 2(5), 474-489. doi:10.1007/bf00056743

Benavides, J. (2013). Perturbaciones en las turberas de páramo: la acción del hombre y el clima. En J. Cortés-Duque \& C. Sarmiento. (Eds.), Visión socio ecosistémica de los páramos y la alta montaña colombiana: memorias del proceso de definición de criterios para la delimitación de páramos (pp. 81-87). Bogotá, D.C., Colombia: Instituto de Investigación de Recursos Biológicos Alexander von Humboldt.

Castro, M. (2005). Complejo turbera de la Chonta. En X. Izurieta. (Ed.), Turberas Altoandinas. Espacios Frágiles de Vida y Cultura (pp. 1-6). Quito, Ecuador: Global Peatland Initiative/NC-IUCN/ ECOPAR/GRUPO PARAMO.

Secretaría de la Convención de Ramsar. (2007). Manual 14. Turberas (lineamientos para la acción mundial sobre las turberas). Gland, Suiza: Secretaría de la Convención de Ramsar.

Chimner, R., \& Ewel, K. (2005). A Tropical Freshwater Wetland: II. Production, Decomposition, and Peat Formation. Wetlands Ecology And Management, 13(6), 671-684. doi: 10.1007/s11273-005-0965-9

Hooghiemstra, H., Cleef, A., Noldus, C., \& Kappelle, M. (1992). Upper Quaternary vegetation dynamics and palaeoclimatology of the La Chonta bog area (Cordillera de Talamanca, Costa Rica). Journal Of Quaternary Science, 7(3), 205-225. doi:10.1002/jqs.3390070303

Horich, C. (1959). The Home of Blechnum Buchtienii in Costa Rica. American Fern Journal, 49(2), 69. doi: 10.2307/1545924

Horn, S., \& Clark, L. (1992). Pollen Viability in Chusquea subtessellata (Poaceae: Bambusoideae). Biotropica, 24(4), 577. doi: $10.2307 / 2389024$

Iturraspe, R. (2010). Las turberas de Tierra del Fuego y el cambio climático global. Buenos Aires, Argentina: Fundación Humedales / Wetlands International.

Jiménez, J. A. (2016). Bogs, Marshes, and Swamps of Costa Rica en Costa Rican Ecosystems. En: M. Kappelle. (Ed.), Costa Rican Ecosystems (pp. 683-705). Chicago, USA: The University of Chicago Press.

Kappelle, M. (1996). Los Bosques de Roble (Quercus) de la Cordillera de Talamanca, Costa Rica: Biodiversidad, Ecología, Conservación y Desarollo. Santo Domingo de Heredia, Costa Rica: Instituto Nacional de Biodiversidad (INBio).

Kappelle, M., \& Horn, S. P. (2016). The Paramo ecosystem of Costa Rica's highlands. En M. Kappelle. (Ed.), Costa Rican Ecosystems (pp. 492-523). Chicago, USA: The University of Chicago Press.

Martínez, A., Pontevedra, X., Novoa, J. C., Rodríguez, R., \& LópezSáez, J. A. (2009). En A. Martínez. (Coord.), Turberas ácidas de esfagnos Bases ecológicas preliminares para la conservación de los tipos de hábitat de interés comunitario en España (pp.9-16). Madrid, España: Ministerio de Medio Ambiente, y Medio Rural y Marino.

Obando, L., \& Soto, G. (1993). La turbera del Río Silencio (El Cairo, Siquirres, Costa Rica): paleoambientes lagunares influenciados por las cenizas del volcán Turrialba. Revista Geológica De América Central, 15, 41-48. doi: 10.15517/ rgac.v0i15.13236

Pontevedra, X. (2001). Antecedentes de los estudios de turberas. En A. Martínez y E. García-Rodeja. (Coord.), Turberas de montaña de Galicia (pp. 17-30). Galicia, España: Consejería de Medio Ambiente.

Programa Nacional de Humedales SINAC-MINAE. (2002). Ficha técnica turberas de Talamanca. San José, Costa Rica: SINAC-MINAE.

See Digital Appendix at: / Ver Apéndice digital en: http://investiga.uned.ac.cr/revistas/index.php/cuadernos 